T. Sekiguchi

Nagoya Math. J.

Vol. 88 (1982), 197-212

\title{
ON THE FIELDS OF RATIONALITY FOR CURVES AND FOR THEIR JACOBIAN VARIETIES
}

\author{
TSUTOMU SEKIGUCHI
}

\section{Introduction}

Throughout the paper, a scheme means a noetherian scheme. By a curve $C$ over a scheme $S$ of genus $g$, we mean a proper and smooth $S$ scheme with irreducible curves of genus $g$ as geometric fibres. In the previous paper [15], the author showed that the field of moduli for a nonhyperelliptic curve over a field coincides with the one for its canonically polarized jacobian variety, and in [16], he gave a partial result on the coincidence of the fields of rationality for a hyperelliptic curve and for its canonically polarized jacobian variety. In the present paper, we will discuss the isomorphy of the isomorphism schemes of two curves over a scheme and of their canonically polarized jacobian schemes, by using Oort-Steenbrink's result [12]. Our result is as follows:

Let $C$ and $C^{\prime}$ be two curves over a scheme $S, P(C)$ and $P\left(C^{\prime}\right)$ be their canonically polarized jacobian schemes, respectively. If $C$ and $C^{\prime}$ are nonhyperelliptic, the canonical map

$$
\operatorname{Isom}_{S}\left(C, C^{\prime}\right) \longrightarrow \operatorname{Isom}_{S}\left(P\left(C^{\prime}\right), P(C)\right) /\{ \pm 1\}
$$

is isomorphic. If $C$ and $C^{\prime}$ are hyperelliptic and $S$ is a scheme over $\operatorname{Spec}(Z[1 / 2])$, the canonical map

$$
\operatorname{Isom}_{S}\left(C, C^{\prime}\right) \longrightarrow \operatorname{Isom}_{S}\left(P\left(C^{\prime}\right), P(C)\right)
$$

is isomorphic.

As a corollary to this result, we get the following:

Let $C$ be a non-hyperelliptic curve over a field of any characteristic or $a$ hyperelliptic curve over a field of characteristic $\neq 2$. If the polarized jacobian variety $P(C)$ is rational over a field $k$, then $C$ is also rational over $k$ and vice versa. In particular, the field of moduli for $C$ coincides with 
the one for $P(C)$.

We will start with reviewing Oort-Steenbrink's result in $\S 1$, and in $\S 2$, we will discuss the isomorphism schemes of curves and of polarized abelian schemes. In $\S 3$, we will state the result about the fields of rationality for curves. Lastly, as an appendix, we will give an estimation of possible prime factors of the order of an automorphism of an abelian variety, which is a transfer of one of Homma's results [5] on curves to the case of abelian varieties.

\section{§ 1. Oort-Steenbrink's result}

Let $M_{g, n}$ (resp. $A_{g, 1, n}$ ) be the moduli scheme of curves of genus $g$ (resp. of principally polarized abelian varieties of dimension g) with level $n$-structures (in the sense of [14]). According to the works of Mumford and Popp (cf. [9] and [14]), the following is known.

Theorem 1.1. When $n \geqq 3, M_{g, n}$ and $A_{g, 1, n}$ are fine moduli schemes, and they are smooth and quasi-projective over $\operatorname{Spec}(Z[1 / n])$.

For a positive integer $m$, we put $M_{g, n}^{(m)}=M_{g, n} \otimes Z[1 / m n]$ and $A_{g, 1, n}^{(m)}=$ $A_{g, 1, n} \otimes Z[1 / m n]$. Let $J_{n}: M_{g, n} \rightarrow A_{g, 1, n}$ be the Torelli map defined by $(C, \alpha)$ $\rightarrow(P(C), \alpha)$, where $C$ is a curve of genus $g, P(C)=(J(C), \lambda(C))$ is the canonically polarized jacobian variety of $C$ and $\alpha: J(C)_{n} \rightarrow(Z / n Z)^{2 g}$ is a level $n$-structure on $C$. Now, let $G$ be the constant group scheme consisting of $\{ \pm 1\}$. We define an action of $G$ on $M_{g, n}$ by $(C, \alpha) \rightarrow(C,-\alpha)$. Then obviously there exists a geometric quotient $V_{g, n}=M_{g, n} / G$, and $J_{n}$ induces a morphism $\iota: V_{g, n} \rightarrow A_{g, 1, n}$.

Definition 1.2. A curve $C$ over a scheme $S$ is said to be hyperelliptic, when there exists an $S$-involution $\sigma$ of $C$ such that $C /\langle\sigma\rangle$ is a curve of genus 0 over $S$.

As for hyperelliptic curves, Laudal-L $\phi$ nsted's result [7] asserts,

Theorem 1.3. (Laudal-L $\phi$ nsted). Let $n \geqq 3$. Then there exists a fine moduli $\operatorname{Spec}(Z[1 / n])$-scheme $H P_{g_{n}}$ for hyperelliptic curves of genus $g$ with level $n$-structures. And it is precisely attained by a closed subscheme of $M_{g, n}$ which is smooth of relative dimension $2 g-1$ over $\operatorname{Spec}(Z[1 / n])$.

Under these notations, Oort-Steenbrink's result [12] is formulated as follows: 
Theorem 1.4 (Oort-Steenbrink). Let $n \geqq 3$. Then $\iota: V_{g, n}^{(2)} \rightarrow A_{g, 1, n}^{(2)}$ and $J_{n}: H P_{g, n}^{(2)} \rightarrow A_{g, 1, n}^{(2)}$ are locally closed immersions. In particular, even if characteristic 2, ८ is a locally closed immersion at every non-hyperelliptic point.

Definition 1.5. We say a polarized abelian variety $(X, \lambda)$ is decomposable, when there exist polarized abelian varieties $\left(X_{i}, \lambda_{i}\right)(i=1,2)$ and an isomorphism $\left(X_{1} \times X_{2}, \lambda_{1} \times \lambda_{2}\right) \cong(X, \lambda)$. When $(X, \lambda)$ is not decomposable, we say that it is indecomposable.

The following is an elementary criterion for decomposability of a principally polarized abelian variety, which was settled during a conversation with Dr. R. Sasaki.

Lemma 1.6. A principally polarized abelian variety $(X, \lambda)$ over a field is decomposable if and only if there exist effective divisors $D_{1}$ and $D_{2}$ such that $\lambda=\phi_{D_{1}+D_{2}}$, where $\phi_{D}: X \rightarrow \hat{X}$ is the homomorphism defined by $x \mapsto$ $T_{x}^{*} \mathcal{O}_{X}(D) \otimes \mathcal{O}_{X}(-D)$ for a divisor $D$ on $X$.

Proof. Since the "only if" part is obvious, it suffices to show the "if" part. Let $K_{0}\left(D_{i}\right)$ be the connected component of $\operatorname{Ker}\left(\phi_{D_{i}}\right)$ containing the origin for each $i=0,1$. Since $K_{0}\left(D_{0}\right) \cap K_{0}\left(D_{1}\right) \subset \operatorname{Ker}\left(\phi_{D_{0}+D_{1}}\right)=\operatorname{Ker}(\lambda)=\{0\}$, the canonical map $\iota: K_{0}\left(D_{0}\right) \times K_{0}\left(D_{1}\right) \rightarrow X$ is injective. So we have only to check the equality

$$
\operatorname{dim} K_{0}\left(D_{0}\right)+\operatorname{dim} K_{0}\left(D_{1}\right)=\operatorname{dim} X .
$$

Now we put $\operatorname{dim} X=g, \operatorname{dim} K_{0}\left(D_{i}\right)=g_{i}$ for $i=0,1$ and $D=D_{0}+D_{1}$. Then the polynomial $P_{i}(n)$ defined by the Euler-Poincaré characteristic $\chi\left(\mathcal{O}_{X}\left(D_{i}+n D\right)\right)$ has $g_{i}$ as the multiplicity of $n=0$ (cf. Kempf's appendix to [10]). Hence, by Riemann-Roch theorem, $P_{i}(n)$ has the leading term

$$
\frac{1}{g !}\left(\begin{array}{l}
g \\
g_{i}
\end{array}\right)\left(D_{\imath}^{g-g_{i i}} \cdot D_{1-i}^{g_{i}}\right) \cdot n^{g_{i}},
$$

where $\left(D_{i}^{g-g_{i}} \cdot D_{1-i}^{g_{i}}\right)$ means the intersection number. On the other hand, by using $\ell$-adic representation, we have

$$
\begin{aligned}
P_{i}(n)^{2} & =\chi\left(\mathcal{O}_{X}\left(D_{i}+n D\right)\right)^{2}=\operatorname{deg}\left(\phi_{D_{i+n D}}\right) \\
& =\operatorname{deg}\left(\phi_{D}^{-1} \circ \phi_{D_{i}+n D}\right)=\operatorname{det} T_{\ell}\left(\phi_{D}^{-1} \circ \phi_{D_{i}+n D}\right) .
\end{aligned}
$$

Here note that $\phi_{D}^{-1} \circ \phi_{D_{i}+n D} \in \operatorname{End}(X)$. Hence $P_{i}(n)^{2}$ has rational】integral coefficients (cf. [11], Chap. IV, §19, Theorem 4); in particular, 


$$
\frac{1}{g !}\left(\begin{array}{l}
g \\
g_{i}
\end{array}\right)\left(D_{i}^{g-g_{i}} \cdot D_{1-i}^{g_{i}}\right)
$$

is integral. Moreover, $\mathbf{\text { since }}$

$$
1=\chi\left(\mathcal{O}_{X}(D)\right)=\left(D^{g}\right) / g !=\frac{1}{g !} \sum_{j=0}^{g}\left(\begin{array}{c}
g \\
j
\end{array}\right)\left(D^{g-j} \cdot D^{j}\right),
$$

we get

$$
\left(\begin{array}{l}
g \\
j
\end{array}\right)\left(D_{0}^{g-j} \cdot D_{0}^{j}\right)= \begin{cases}1 & \text { if } j=g_{0} \\
0 & \text { if } j \neq g_{0}\end{cases}
$$

and

$$
g_{1}=g-g_{0}
$$

Q.E.D.

Obviously the points of $A_{g, 1, n}$ corresponding to indecomposable abelian varieties form a Zariski open subset, which we denote by $U_{\theta, 1, n}$. Then, noting the compatibility of the specializations of a curve and of its polarized jacobian variety (cf. [13], Lemma 6 or [15], Proposition 4.1), we get,

CoROLLARY 1.7. If $n \geqq 3$,

$$
\iota: V_{g, 1, n}^{(2)} \longrightarrow U_{g, 1, n}^{(2)}
$$

and

$$
J_{n}: H P_{g, n}^{(2)} \longrightarrow U_{g, 1, n}^{(2)}
$$

are closed immersions.

\section{§2. Isomorphism schemes of curves and abelian varieties}

Let $S$ be a scheme, $C, C^{\prime}$ be two curves over $S$, and $P, P^{\prime}$ be two polarized abelian schemes over $S$. Then by Grothendieck's theory in FGA, there exist isomorphism schemes

$$
\operatorname{Isom}_{S}\left(C, C^{\prime}\right) \longrightarrow S
$$

and

$$
\operatorname{Isom}_{S}\left(P^{\prime}, P\right) \longrightarrow S
$$

representing the functors from the category of $S$-schemes to the category 
of sets:

$$
T \longmapsto \operatorname{Isom}_{T}\left(C \times{ }_{S} T, C^{\prime} \times{ }_{S} T\right)
$$

and

$$
T \longmapsto \operatorname{Isom}_{T}\left(P^{\prime} \times{ }_{S} T, P \times{ }_{S} T\right),
$$

respectively. These schemes have the following property:

TheOREm 2.1. $\operatorname{Isom}_{S}\left(C, C^{\prime}\right)$ and $\operatorname{Isom}_{S}\left(P^{\prime}, P\right)$ are finite and unramified over $S$.

The proof for curves is given by ([1], Theorem 1.11) and the one for polarized abelian varieties follows similarly (cf. [16], Theorem 3.1).

Hereafter, we will discuss a relation between the isomorphisms of curves and those of their polarized jacobian varieties. Now, let $J: \mathbf{I s o m}_{S}$ $\left(C, C^{\prime}\right) \rightarrow \operatorname{Isom}_{S}\left(P\left(C^{\prime}\right), P(C)\right)$ be the morphism defined by $\sigma \mapsto \operatorname{Pic}^{0}(\sigma)$ for $\sigma \in \operatorname{Isom}_{S}\left(C, C^{\prime}\right)$. The next is a well-known result.

THEOREm 2.2. Let $C$ and $C^{\prime}$ be two curves over an algebraically closed field $k$. Then we have

(i ) $J: \operatorname{Isom}_{k}\left(C, C^{\prime}\right) \rightarrow \operatorname{Isom}_{k}\left(P\left(C^{\prime}\right), P(C)\right)$ is injective;

(ii) if $C$ and $C^{\prime}$ are non-hyperelliptic, the canonically induced map $\bar{J}: \operatorname{Isom}_{k}\left(C, C^{\prime}\right) \rightarrow \operatorname{Isom}_{k}\left(P\left(C^{\prime}\right), P(C)\right) /\{ \pm 1\}$ is a bijection;

(iii) if $C$ and $C^{\prime}$ are hyperelliptic, $J: \operatorname{Isom}_{k}\left(C, C^{\prime}\right) \rightarrow \operatorname{Isom}_{k}\left(P\left(C^{\prime}\right), P(C)\right)$ is a bijection (cf. [8] or [17]).

Combining these theorems, we obtain the following:

Proposition 2.3. $J: \operatorname{Isom}_{S}\left(C, C^{\prime}\right) \rightarrow \operatorname{Isom}_{S}\left(P\left(C^{\prime}\right), P(C)\right)$ is a closed immersion.

Proof. Theorem 2.2 implies that $J$ is radicial. On the other hand, by using (EGA II, (5.4.3)) and (EGA IV,$(17.1 .3)$ ), we can deduce easily the fact that $J$ is proper and unramified. Hence, $J$ is a closed immersion.

Q.E.D.

Theorem 2.4. Let $C$ and $C^{\prime}$ be two hyperelliptic curves over $S$. Assume that $S$ is a scheme over $\operatorname{Spec}(Z[1 / 2])$. Then

$$
J: \operatorname{Isom}_{S}\left(C, C^{\prime}\right) \longrightarrow \operatorname{Isom}_{S}\left(P\left(C^{\prime}\right), P(C)\right)
$$

is an isomorphism. 
Proof. It suffices to show our assertion for a suitably small open neighbourhood at every point of $S$. So we may exchange $S$ for a smaller one.

Let $H_{g, 1, n}$ be the fine moduli space of the principally polarized abelian varieties with linear rigidifications and with level $n$-structures (cf. [9]). Then $H_{g, 1,4}$ is a principal covering of $H_{g, 1,1}^{(2)}$ with Galois group $\operatorname{Sp}(g, Z / 4 Z)$ (cf. [9], Lemma 7.11). Let $\pi: H_{g, 1,4} \rightarrow H_{g, 1,1}^{(2)}$ be the canonical map. If we take $S$ to be suitably small, $P\left(C^{\prime}\right)$ has a linear rigidification. Hence $P\left(C^{\prime}\right)$ with this linear rigidification defines an $S$-valued point

$$
u: S \longrightarrow H_{g, 1,1}^{(2)}
$$

(n.b., we have assumed that $S$ is over $\operatorname{Spec}(Z[1 / 2]))$. Since $\pi$ is étale, i : we take the fibre product $S^{\prime}=S \times_{H_{g, 1,1}^{(2)}} H_{g, 1,4}$ :

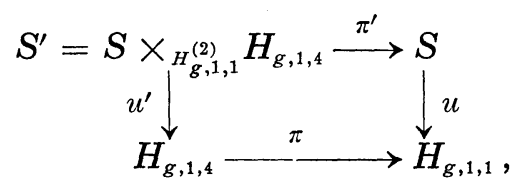

$S^{\prime}$ becomes an étale, a fortiori, faithfully flat covering of $S$. Since $H_{g, 1,4}$ is a fine moduli space, $u^{\prime}$ determine a level 4-structure $\alpha$ of $C_{S^{\prime}}^{\prime}=C^{\prime} \times{ }_{S} S^{\prime}$ $\rightarrow S^{\prime}$. So $C_{S^{\prime}}^{\prime}$ with $\alpha$ defines an $S^{\prime}$-valued point

$$
a: S^{\prime} \longrightarrow M_{g, 4} \text {. }
$$

Here $a$ factors through the hyperelliptic locus $H P_{g, 4}$, for we have assumed that $C^{\prime}$ is hyperelliptic.

Now we take any $S^{\prime}$-scheme $f: S^{\prime \prime} \rightarrow S^{\prime}$ and any $S^{\prime \prime}$-valued point $\tau$ of Isom $_{S^{\prime}}\left(P\left(C_{S^{\prime}}^{\prime}\right), P\left(C_{S^{\prime}}\right)\right)$. Then $\tau\left(\alpha \times{ }_{S^{\prime}} S^{\prime \prime}\right)$ is a level 4-structure of $P\left(C_{S^{\prime}}\right)$ $\times_{S^{\prime}} S^{\prime \prime}=P\left(C_{S^{\prime \prime}}\right)$, and $\left(C_{S^{\prime \prime}}, \tau\left(\alpha \times_{S^{\prime}} S^{\prime \prime}\right)\right)$ determines an $S^{\prime \prime}$-valued point $b$ of $H P_{g, 4}$. Since $\tau$ induces an isomorphism between $\left(P\left(C_{S^{\prime \prime}}^{\prime}\right), \alpha \times_{S^{\prime}} S^{\prime \prime}\right)$ and $\left(P\left(C_{S^{\prime \prime}}\right), \tau\left(\alpha \times_{S^{\prime}} S^{\prime \prime}\right)\right)$, we get the equality

$$
J_{4}(a \circ f)=J_{4}(b) \text {. }
$$

Hence, by Oort-Steenbrink's result Theorem 1.4, we have

$$
a \circ f=b,
$$

that is, there exists an isomorphism

$$
\phi:\left(C_{S^{\prime \prime}}, \tau\left(\alpha \times_{S^{\prime}} S^{\prime \prime}\right)\right) \longrightarrow\left(C_{S^{\prime \prime}}^{\prime \prime}, \alpha \times_{S^{\prime}} S^{\prime \prime}\right) .
$$


Since $J(\phi)$ induces the isomorphism between level 4-structures $\alpha \times{ }_{S^{\prime}} S^{\prime \prime}$ and $\tau\left(\alpha \times{ }_{s^{\prime}} S^{\prime \prime}\right)$, by Serre's theorem (cf. [11], Chap. IV, § 21, Theorem 5), we have

$$
J(\phi) \times{ }_{s^{\prime \prime}} \operatorname{Spec}\left(k\left(s^{\prime \prime}\right)\right)=\tau \times{ }_{s^{\prime \prime}} \operatorname{Spec}\left(k\left(s^{\prime \prime}\right)\right)
$$

for every point $s^{\prime \prime}$ of $S^{\prime \prime}$. Hence, by the rigidity (cf. [9], Corollary 6.2), we get the equality

$$
J(\phi)=\tau .
$$

This implies the surjectivity of

$$
J: \operatorname{Isom}_{S^{\prime \prime}}\left(C_{S^{\prime \prime}}, C_{S^{\prime \prime}}^{\prime}\right) \longrightarrow \operatorname{Isom}_{S^{\prime \prime}}\left(P\left(C_{S^{\prime \prime}}^{\prime}\right), P\left(C_{S^{\prime \prime}}\right)\right),
$$

and combining with Proposition 2.3, we obtain the isomorphy of

$$
J_{S^{\prime}}: \operatorname{Isom}_{S^{\prime}}\left(C_{S^{\prime}}, C_{S^{\prime}}^{\prime}\right) \longrightarrow \operatorname{Isom}_{S^{\prime}}\left(P\left(C_{S^{\prime}}^{\prime}\right), P\left(C_{S^{\prime}}\right)\right) .
$$

Moreover, since $S^{\prime}$ is faithfully flat over $S$, by using the descent theory (cf. EGA IV ${ }^{2}$, Proposition (2.7.1)), we get the isomorphy of

$$
J: \operatorname{Isom}_{S}\left(C, C^{\prime}\right) \longrightarrow \operatorname{Isom}_{S}\left(P\left(C^{\prime}\right), P(C)\right) \text {. }
$$

Q.E.D.

The similar result for non-hyperelliptic curves is also established in a similar way as above.

THEOREM 2.5. Let $C$ and $C^{\prime}$ be two non-hyperelliptic curves (i.e., every geometric fibre of $C$ and $C^{\prime}$ is non-hyperelliptic). Let $G$ be the constant group scheme consisting of $\{ \pm 1\}$. Obviously $G$ acts naturally on Isom $_{s}$ $\left(P\left(C^{\prime}\right), P(C)\right)$ and the geometric quotient $\operatorname{Isom}_{S}\left(P\left(C^{\prime}\right), P(C)\right) / G$ exists. Then the natural map

$$
\bar{J}: \mathbf{I s o m}_{S}\left(C, C^{\prime}\right) \longrightarrow \operatorname{Isom}_{S}\left(P\left(C^{\prime}\right), P(C)\right) / G
$$

is an isomorphism.

Proof. For brevity we put $\boldsymbol{I}=\mathbf{I s o m}_{S}\left(C, C^{\prime}\right)$ and $\left.\boldsymbol{J}=\mathbf{I}_{\mathbf{s o m}_{S}}\left(P\left(C^{\prime}\right)\right), P(C)\right)$. First we note that $G$ acts freely on the open subset $N_{g, n}$ consisting of non-hyperelliptic points in $M_{g, n}(n \geqq 3)$ and on $J$, respectively. Hence the canonical maps $N_{g, n} \rightarrow N_{g, n} / G$ and $\boldsymbol{J} \stackrel{\pi}{\rightarrow} \bar{J}=J / G$ are principal homogeneous fibre bundles. In view of Theorems 2.1 and 2.2, we get that $\bar{J}$ is proper, radicial and unramified, and so a closed immersion. 
Now, in a similar way as in the proof of Theorem 2.4, there exist an open neighbourhood at each point of $S$, which we denote also by $S$, and a faithfully flat covering $S^{\prime} \rightarrow S$, such that $S$ is a scheme over $\operatorname{Spec}(Z[1 / n])$ for some $n \geqq 3$ and $P\left(C_{S^{\prime}}^{\prime}\right)$ has a level $n$-structure $\alpha$. Let $S^{\prime \prime}$ be any $S^{\prime}$ scheme, and $\bar{\tau}$ be any $S^{\prime \prime}$-valued point of $J$. Since $\pi: J \rightarrow J$ is faithfully flat, if we take the fibre product

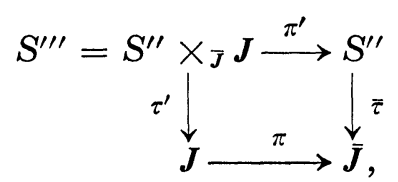

$\pi^{\prime}: S^{\prime \prime \prime} \rightarrow S^{\prime \prime}$ is also faithfully flat. Now $\tau^{\prime}$ defines an isomorphism

$$
\tau^{\prime}:\left(P\left(C_{S^{\prime}}^{\prime}\right) \times{ }_{S^{\prime}} S^{\prime \prime \prime}, \alpha \times{ }_{S^{\prime}} S^{\prime \prime \prime}\right) \longrightarrow\left(P\left(C_{S^{\prime}}\right) \times{ }_{S^{\prime}} S^{\prime \prime \prime}, \tau^{\prime}\left(\alpha \times{ }_{S^{\prime}} S^{\prime \prime \prime}\right)\right) .
$$

Hence, by using Theorem 1.4 and Serre's theorem as in the proof of Theorem 2.4, we obtain an isomorphism

$$
\sigma^{\prime}:\left(C_{S^{\prime \prime \prime}, \nu \tau}\left(\alpha \times{ }_{S^{\prime}} S^{\prime \prime \prime}\right)\right) \stackrel{\sim}{\longrightarrow}\left(C_{S^{\prime \prime \prime}}^{\prime}, \alpha \times{ }_{S^{\prime}} S^{\prime \prime \prime}\right)
$$

with an $S^{\prime \prime \prime}$-valued point $\nu$ of $G$, and the equality

$$
J\left(\sigma^{\prime}\right)=\nu \tau
$$

Here we consider the following commutative diagram:

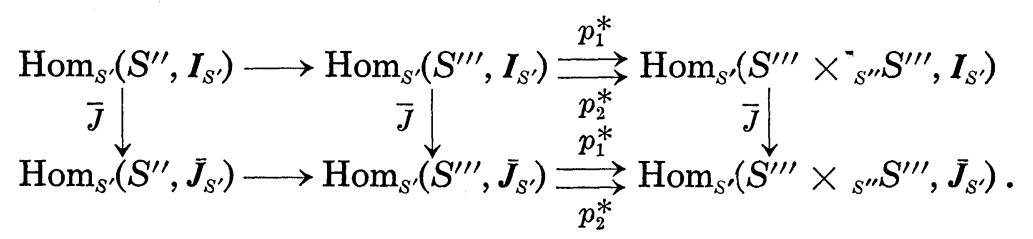

According to the descent theory (cf. SGA 1, Ex. VIII, Theorem 5.2), the horizontal lines are exact. By our construction of $\sigma^{\prime}$,

$$
\bar{J}\left(p_{1}^{*} \sigma^{\prime}\right)=p_{1}^{*} \pi\left(\tau^{\prime}\right)=p_{2}^{*} \pi\left(\tau^{\prime}\right)=\bar{J}\left(p_{2}^{*} \sigma^{\prime}\right) .
$$

Since $\bar{J}$ is a monomorphism, we get the equality

$$
p_{1}^{*} \sigma^{\prime}=p_{2}^{*} \sigma^{\prime}
$$

Therefore, there exists $\sigma \in \operatorname{Hom}_{S^{\prime}}\left(S^{\prime}, I\right)$ which induces $\sigma^{\prime}$, and so

$$
\bar{J}(\sigma)=\bar{\tau} .
$$

Thus we obtain the bijectivity of 


$$
\bar{J}: \operatorname{Hom}_{S^{\prime}}\left(S^{\prime \prime}, I_{S^{\prime}}\right) \longrightarrow \operatorname{Hom}_{S^{\prime}}\left(S^{\prime \prime}, \bar{J}\right),
$$

and the isomorphy of

$$
\bar{J}: I_{S^{\prime}} \longrightarrow J_{S^{\prime}}
$$

Once more using the descent theory, we obtain the fact that $\bar{J}$ is isomorphic over $S$, and we are done.

Lastly, we note the fact that if $P_{1}, P_{2}$ and $P_{3}$ are polarized abelian schemes over $S$, then the composition morphism

$$
\circ: \operatorname{Isom}_{S}\left(P_{1}, P_{2}\right) \times \operatorname{Isom}_{S}\left(P_{2}, P_{3}\right) \longrightarrow \operatorname{Isom}_{S}\left(P_{1}, P_{3}\right)
$$

induces naturally a morphism

$$
\bar{\circ}: \operatorname{Isom}_{S}\left(P_{2}, P_{3}\right) / G \times \operatorname{Isom}_{S}\left(P_{1}, P_{2}\right) / G \longrightarrow \operatorname{Isom}_{S}\left(P_{1}, P_{3}\right) / G .
$$

\section{§3. Fields of rationality for curves}

We will discuss in this paragraph a relation between the fields of rationality for a curve and for its jacobian variety as an application of the result in the preceding paragraph.

TheOREM 3.1. Let $S$ be a scheme over $\operatorname{Spec}(Z[1 / 2])$, and $S^{\prime}$ be a faithfully flat $S$-scheme. Let $C^{\prime}$ be a hyperelliptic curve over $S^{\prime}$ and $P$ be a principally polarized abelian scheme over $S$ such that there exists an isomorphism

$$
\phi^{\prime}: P\left(C^{\prime}\right) \stackrel{\sim}{\longrightarrow} \times{ }_{s} S^{\prime} .
$$

Then there exist a curve $C$ over $S$ and isomorphisms

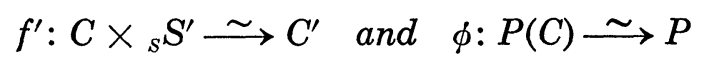

such that

$$
\phi_{S^{\prime}} \circ J\left(f^{\prime}\right)=\phi^{\prime} .
$$

The same result can be obtained for non-hyperelliptic curves.

TheOREM 3.2. Let $S^{\prime}$ be a faithfully flat $S$-scheme, $C^{\prime}$ be a nonhyperelliptic curve over $S^{\prime}$ (i.e., $C^{\prime}$ has non-hyperelliptic curves as geometric fibres), and $P$ be a principally polarized abelian scheme over $S$ having an isomorphism

$$
\phi^{\prime}: P\left(C^{\prime}\right) \stackrel{\sim}{\longrightarrow} \times{ }_{s} S^{\prime}
$$


Then there exist a curve $C$ over $S$ and isomorphisms

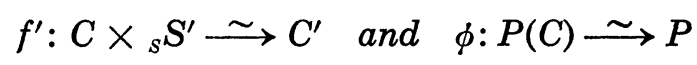

such that

$$
\phi_{S^{\prime}} \circ J\left(f^{\prime}\right)=\phi^{\prime}
$$

The proofs of these theorems can be done in a similar way. So we will give only the one of the latter.

Proof of Theorem 3.2. We put $S^{\prime \prime}=S^{\prime} \times{ }_{s} S^{\prime}$ and $p_{i}: S^{\prime \prime} \rightarrow S^{\prime}$ : the projections to the $i$-th component for $i=1,2$. For short, we put $I^{\prime}=$ $\operatorname{Isom}_{S^{\prime \prime}}\left(p_{1}^{*} C, p_{2}^{*} C^{\prime}\right), J^{\prime}=\operatorname{Isom}_{S^{\prime \prime}}\left(p_{2}^{*} P\left(C^{\prime}\right), p_{1}^{*} P\left(C^{\prime}\right)\right)$ and $\bar{J}^{\prime}=J^{\prime} / G$, where $G$ $=\{ \pm 1\}$. By our assumption, there exists a descent deta $\phi \in \operatorname{Hom}_{S^{\prime \prime}}\left(S^{\prime \prime}, J^{\prime}\right)$; that is, it satisfies the following:

$$
\Delta^{*} \phi=1_{P\left(C^{\prime}\right)} \text { and } p_{13}^{*} \phi=p_{12}^{*} \phi \circ p_{23}^{*} \phi,
$$

where $\Delta: S^{\prime} \rightarrow S^{\prime \prime}=S^{\prime} \times{ }_{s} S^{\prime}$ is the diagonal morphism and $p_{i j}: S^{\prime} \times{ }_{s} S^{\prime}$ $\times{ }_{S} S^{\prime} \rightarrow S^{\prime \prime}$ is the projection to the $(i, j)$-th component. Therefore, $\pi(\phi)=$ $\bar{\phi} \in \operatorname{Hom}_{S^{\prime}}\left(S^{\prime \prime}, \bar{J}^{\prime}\right)$ satisfies the same condition

$$
\Delta^{*} \bar{\phi}=\overline{\mathbf{1}}_{P\left(C^{\prime}\right)} \quad \text { and } \quad p_{13}^{*} \bar{\phi}=p_{12}^{*} \bar{\phi} \circ p_{23}^{*} \bar{\phi} .
$$

Hence, by virtue of Theorem 2.5, we get an isomorphism $\sigma \in \operatorname{Hom}_{S^{\prime \prime}}\left(S^{\prime \prime}, I^{\prime}\right)$ satisfying the condition

$$
\Delta^{*} \sigma=\mathbf{1}_{C^{\prime}} \text { and } p_{13}^{*} \sigma=p_{23}^{*} \sigma \circ p_{12}^{*} \sigma .
$$

Thus, by using (SGA 1, Ex. VIII, Proposition 7.8), we can descent $C^{\prime}$ over $S$.

Q.E.D.

As direct consequences of Theorems 3.1 and 3.2, we obtain the following corollary.

CoRollaries 3.3. Let $k$ be a field of characteristic $p$, and $k_{0}$ be $a$ subfield of $k$. Let $C$ be a hyperelliptic curve over $k$ with $p \neq 2$ or a nonhyperelliptic curve over $k$ with any $p$. If there exists a principally polarized abelian variety $P$ over $k_{0}$ such that $P \otimes_{k_{0}} k \simeq P(C)$, there exists a curve $C_{0}$ over $k_{0}$ such that $C_{0} \otimes_{k_{0}} k \simeq C$. In particular, the field of moduli for $C$ coincides with the one for $P(C)$.

Appendix. Prime orders of an automorphism of an abelian variety 
We begin this appendix by transferring one of Homma's results [5] on curves to the case of abelian varieties.

Proposition A1. Let $X$ be an abelian variety of dimension $g$ over an algebraically closed field of any characteristic, and $\lambda: X \rightarrow \hat{X}$ be a principal polarization.

(1) Let $\sigma$ be an automorphism of $X$ of prime order $q$. Then we have

(i) $q \leqq 2 g+1$;

(ii) if we denote by $P(n)$ the polynomial defined by $\operatorname{deg}\left(\sigma-n \mathbf{1}_{X}\right)$, $2 \operatorname{dim}(\operatorname{Ker}(\sigma-1))=$ the multiplicity of 1 as a zero of $P(n)$;

(iii) if $q=2 g+1, \operatorname{dim}(\operatorname{Ker}(\sigma-1))=0$;

(iv) if $\operatorname{dim}(\operatorname{Ker}(\sigma-1))=0$ (in particular, if $X$ is simple), $q=(2 g / r)+1$ for some positive integer $r$.

(2) Assume that $(X, \lambda)$ is indecomposable (cf. Definition 1.5). Let $\sigma$ be an automorphism of $(X, \lambda)$ or prime order $q$. Then we have

$$
q=2 g+1 \text { or } q \leqq g+1 .
$$

Remark. The estimation (1), (i) is more or less a well-known fact.

Proof. Let $P(n)$ be the polynomial defined by $\operatorname{deg}\left(\sigma-n \mathbf{1}_{X}\right)$ as in (1), (ii). Then $P(n)$ is a monic polynomial of degree $2 g$ with integral coefficients, and it is equal to $\operatorname{det}\left(T_{\ell}(\sigma)-n E\right)$, where $T_{\ell}$ means the $\ell$-adic representation and $E$ is the unit matrix (cf. [11], Chap. IV, $\S 19$, Theorem 4). Since $T_{\ell}(\sigma)$ has the prime order $q$, its eigen-values are 1 or primitive $q$-th roots of the unity. So $P(x)$ must be of the following type:

$$
\text { (*) } \quad P(x)=(x-1)^{e}\left(x^{q-1}+x^{q-2}+\cdots+1\right)^{r}
$$

with $e \geqq 0, r \geqq 1$ and $2 g=e+r(q-1)$.

Thus we obtain the first inequality

$$
q \leqq 2 g+1
$$

We put $K=$ the connected component of $\operatorname{Ker}(\sigma-1)$ containing the origin, and $Y=\operatorname{Im}(\sigma-1)$. Let $\varepsilon: K \rightarrow X$ and $\iota: Y \rightarrow X$ be the canonical inclusions. Then $\sigma-1$ factors through $Y$ :

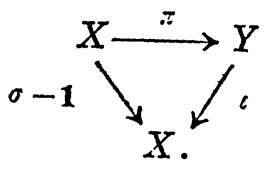


Since $\sigma(\sigma-1)=(\sigma-1) \sigma, \sigma$ acts on $Y$, so we put $\tau=\left.\sigma\right|_{Y}$. Thus we obtain a commutative diagram

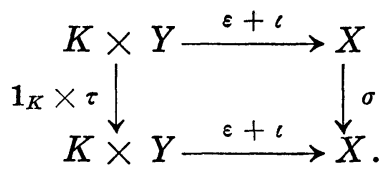

Obviously, $\left(\mathbf{1}+\sigma+\cdots+\sigma^{q-1}\right)(\sigma-1)=0$, and so $\operatorname{Ker}(\sigma-1) \cap Y \subset Y_{q}=$ $\operatorname{Ker}\left(q \mathbf{1}_{Y}\right)$. Hence $\tau-\mathbf{1}_{Y}: Y \rightarrow Y$ and $\varepsilon+\iota: K \times Y \rightarrow X$ are isogenies. Since $\mathbf{1}_{K} \times \tau=(\varepsilon+\iota)^{-1} \sigma(\varepsilon+\iota)$ in $\operatorname{End}^{0}(K \times Y)=\operatorname{End}(K \times Y) \otimes{ }_{Z} Q$, we get

$$
P(x)=(x-1)^{2 \operatorname{dim} K}\left(x^{q-1}+x^{q-2}+\cdots+1\right)^{r} .
$$

This implies that

$$
\operatorname{det}(\tau-1)=q^{r} \text { and } \quad e=2 \operatorname{dim} K .
$$

Thus we obtain the assertion (ii). (iii) and (iv) are direct consequences of $(*)$ and $(* *)$.

Next we will expand the proof of (2). So, hereafter we assume that $\hat{\sigma} \lambda \sigma=\lambda$ and $q<2 g+1$. Then for any $y \in Y$, if we take a point $x \in X$ such that $\pi(x)=y$,

$$
\hat{\varepsilon} \lambda_{c}(y)=\hat{\varepsilon} \lambda_{l} \pi(x)=\hat{\varepsilon} \lambda(\sigma-1)(x)=-\hat{\varepsilon}(\hat{\sigma}-1) \lambda \sigma^{-1}(x)=0 .
$$

That is, $\hat{\varepsilon} \lambda_{\ell}=0$. In particular,

$$
(* * *) \quad \lambda e(Y)=\hat{\pi}(\hat{Y}) .
$$

Dualizing the equality $\hat{\varepsilon} \lambda_{\iota}=0$, we have $\hat{\imath} \lambda \varepsilon=0$. Therefore, if we put $\varepsilon^{*} \lambda$ $=\hat{\varepsilon} \lambda \varepsilon$ and $\iota * \lambda=\hat{\imath} \lambda \iota$, we obtain a commutative diagram

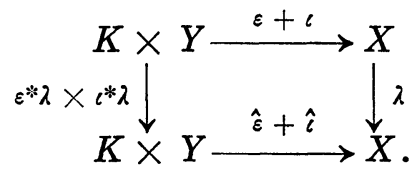

So, noticing our assumption the indecomposability of $(X, \lambda), \varepsilon+\iota$ can not be an isomorphism, that is,

$$
\operatorname{Ker}(\varepsilon+\iota)=K \cap Y \neq\{0\}
$$

Moreover, for $z \in K$, 


$$
\begin{aligned}
\operatorname{Ker}\left(\varepsilon^{*} \lambda\right) \ni z & \Longleftrightarrow \hat{\varepsilon} \lambda \varepsilon(z)=0 \\
& \Longleftrightarrow \lambda \varepsilon(z) \in \operatorname{Ker}(\hat{\varepsilon})=\hat{\pi}(\hat{Y}) \\
& \Longleftrightarrow \varepsilon(z) \in \lambda^{-1}(\hat{\pi} \hat{Y})=\iota(Y)(\text { note }(* * *)) \\
& \Longleftrightarrow z \in K \cap Y .
\end{aligned}
$$

Hence $\operatorname{order}(K \cap Y)=\operatorname{deg}\left(\varepsilon^{*} \lambda\right)$ must be a square of an integer larger than 1 (cf. [11], Chap. IV, $\S 23$, Theorem 4). Since $\operatorname{order}(K \cap Y)$ divides $\operatorname{deg}(\tau-1)$ $=q^{r}$, we get

$$
r \geqq 2 \text {. }
$$

This inequality and $(*)$ yield the inequality

$$
q \leqq g+1
$$

Q.E.D.

As a direct consequence of Proposition A1, we obtain the following:

Corollary A2 (Homma). Let $C$ be a curve of genus $g$ over an algebraically closed field of any characteristic, and $\sigma$ be an automorphism of $C$ of prime order $q$. Then we have

(1) $q=2 g+1$ or $q \leqq g+1$;

(2) if $q=2 g+1$, genus of $C /\langle\sigma\rangle=0$.

In the rest of this appendix, we will discuss an estimation of a power of a prime number dividing the order of the automorphism group of a polarized abelian variety.

Proposition A3. Let $P=(X, \lambda)$ be a polarized abelian variety of dimension $g$ over an algebraically closed field of characteristic $p(\geqq 0)$. Let $q$ be an odd prime integer, and $q^{u}$ be the maximal power of $q$ dividing the order of the automorphism group $\operatorname{Aut}(P)$ of $P$. Then we have

$$
u \leqq \sum_{i \geqq 0}\left[\frac{2 g}{q^{i}(q-1)}\right]
$$

where [ ] means the Gauss symbol.

Proof. We choose a prime integer $\ell$ such that $\ell \neq 2, p$ and $\ell(\bmod q)$ generates the unit group $\left(\boldsymbol{Z} / q^{2} \boldsymbol{Z}\right)^{\times}$of $\boldsymbol{Z} / q^{2} Z$. Then, as is well-known, $\ell$ $\left(\bmod q^{n}\right)$ generates the unit $\operatorname{group}\left(Z / q^{n} Z\right)^{\times}$of $Z / q^{n} Z$. Hence $q^{e}$ is the maximal power of $q$ dividing $\ell^{k}-1$ if and only if $q-1$ divides $k$ and $q^{e-1}$ is the maximal power of $q$ dividing $k$. 
On the other side, by Serre's theorem (cf. [11], Chap. IV, § 21, Theorem 5), the canonical map

$$
\operatorname{Aut}(P) \longrightarrow \operatorname{Aut}\left(X_{\ell}\right) \simeq \mathrm{GL}_{2 g}(Z / \ell Z)
$$

is injective, and the order of $\operatorname{Aut}(P)$ divides the order

$$
\ell^{g(2 g-1)}(\ell-1)\left(\ell^{2}-1\right) \cdots\left(\ell^{2 g}-1\right)
$$

of $\mathrm{GL}_{2 g}(Z \mid \ell Z)$. Therefore, by combining this with the above notice, we get the inequality

$$
u \leqq \sum_{i \geqq 0}(i+1)\left\{\left[\frac{2 g}{q^{i}(q-1)}\right]-\left[\frac{2 g}{q^{i+1}(q-1)}\right]\right\}=\sum_{i \geqq 0}\left[\frac{2 g}{q^{i}(q-1)}\right]
$$

Q.E.D.

Corollary A4. Let $P$ and $q^{u}$ be as in Proposition A3. If $q=2 g+1$, then $u \leqq 1$. If $q=g+1$ or $q=g \geqq 5$, then $u \leqq 2$. If $q=g=3$, then $u \leqq 4$.

Note that in general the estimation in Proposition A3 is best possible. In fact, there exists the following example.

ExAmple. Let $q$ be an odd prime integer, and $\left(Y, \lambda_{Y}\right)$ be a principally polarized abelian variety of dimension $(q-1) / 2$ with an automorphism $\sigma$ of order $q$. Some examples of such polarized abelian varieties $\left(Y, \lambda_{Y}\right)$ are given by Homma [5] as jacobian varieties of some curves, and he determines all such curves. Now we set $X=Y^{r}, \lambda=\Pi \lambda_{Y}: X \rightarrow \hat{X}$ and $P=(X, \lambda)$. Note that $g=\operatorname{dim} X=r \cdot(q-1) / 2$. Let $\sigma_{i}=\mathbf{1}_{Y} \times \cdots \times \mathbf{1}_{Y} \times \sigma^{i} \times \mathbf{1}_{Y} \times \cdots$ $\times \mathbf{1}_{Y} \in \operatorname{Aut}(P)$, and $\mathfrak{S}^{r} \longrightarrow \operatorname{Aut}(P)$ be the inclusion giving the permutations of factors of $X=Y^{r}$, where $\mathfrak{S}_{r}$ is the symmetric group of degree $r$. Moreover, let $A=\left[\left\{S_{r}, \sigma_{i} \mid i=1, \cdots, r\right\}\right] \supset B=\left[\left\{\sigma_{i} \mid i=1, \cdots, r\right\}\right]$ be the subgroups of $\operatorname{Aut}(P)$, generated by $\mathfrak{S}_{r}$ and $\sigma_{i}$ 's. Then obviously $B$ is a normal subgroup of $A$, and we get an exact sequence

$$
1 \longrightarrow B \longrightarrow A \longrightarrow \Im_{r} \longrightarrow 1 \text {. }
$$

Hence, if $q^{u}$ is the maximal power of $q$ dividing the order of $\operatorname{Aut}(P)$,

$$
u \geqq r+\sum_{i \geqq 1} i \cdot\left\{\left[\frac{r}{q^{i}}\right]-\left[\frac{r}{q^{(i+1)}}\right]\right\}=\sum_{i \geqq 0}\left[\frac{2 g}{q^{i}(q-1)}\right],
$$

and so 


$$
u=\sum_{i \geqq 0}\left[\frac{2 g}{q^{i}(q-1)}\right]
$$

Addendum. In the preceding paper ([15], Proposition 4.1), the author discussed the compatibility of the specializations of a curve and its polarized jacobian variety over the same valuation ring. But that proof contained a mistake, and actually there exists a counter example to the compatibility of the specializations of them over the same valuation ring. The author notices here that an essential correction of the proof of ([15], Proposition 4.1) is given by Theorems 3.1 and 3.2.

\section{REFERENCES}

[1] P. Deligne and D. Mumford, The irreducibility of the space of curves of given genus, Publ. Math., 36 (Volume dedicated to O. Zariski), I.H.E.S. (1969), 75-109.

[2] A. Grothendieck, Fondements de la géométrie algébrique, Séminaire Bourbaki, 1952-62, Secrétariat Math., Paris (1962), Refered to as FGA.

[ 3 ] — Eléments de géométrie algébrique (with J. Dieudonne), Pub. Math. I.H.E.S., 1960-67, Refered to as EGA.

[4] A. Grothendieck at al, Séminaire de géométrie algébrique 1, Lecture notes in Math. 224, Springer-Verlag, 1971, Refered to as SGA 1.

[5] M. Homma, Automorphisms of prime order on curves, Manuscripta Math., 33 (1980), 99-109.

[6] S. Koizumi, The fields of moduli for polarized abelian varieties and for curves, Nagoya Math. J., 48 (1972), 37-55.

[ 7 ] O. A. Laudal and K. Lønsted, Deformations of curves I, Moduli for hyperelliptic curves, Algebraic geometry, Proceedings Tromso, Norway 1977, Lecture notes in Math. 687, Springer-Verlag, 1978.

[ 8 ] T. Matsusaka, On a theorem of Torelli, Amer. J. Math., 80 (1958), 784-800.

[ 9 ] D. Mumford, Geometric invariant theory, Ergebnisse, Springer-Verlag, 1965.

[10] — - Varieties defined by quadratic equations, Questioni sulle Varieta Algebriche, Corsi dal C.I.M.E., Edizioni Cremonese, Roma 1969.

[11] —-, Abelian varieties, Tata Inst. Studies in Math., Oxford Univ. Press, London and New York, 1970.

[12] F. Oort and J. Steenbrink, The local Torelli problem for algebraic curves, Univ. Utrecht, Dep. Math., Preprint Nr. 136, 1979.

[13] F. Oort and K. Ueno, Principally polarized abelian varieties of dimension two and three are Jacobian varieties, J. Fac. Sci. Univ. Tokyo, Section IA, Math., 20 (1973), 377-381.

[14] H. Popp, Moduli theory and classification theory of algebraic varieties, Lecture notes in Math. 620, Springer-Verlag, 1977.

[15] T. Sekiguchi, The coincidence of fields of moduli for non-hyperelliptic curves and for their jacobian varieties, Nagoya Math. J., 82 (1981), 57-82.

[16] _ - On the fields of rationality for curves and for abelian varieties, Bull. Fac. Sci. \& Eng. Chuo Univ., 23 (1980) 35-41.

[17] A. Weil, Zum Beweis des Torellischen Satzes, Nachr. Akad. Wissensch. Göttingen, (1957), 33-53. 
Department of Mathematics

Faculty of Science and Engineering

Chuo University

Kasuga, Bunkyo-ku

Tokyo, Japan 